\title{
LATE-ONSET CAPSULAR BAG DISTENSION SYNDROME: A REPORT OF TWO CASES
}

\author{
Paraskevas Zafeiropoulos, Andreas Katsanos, Konstantina Gorgoli, Ioannis Asproudis, Miltiadis Aspiotis
}

University Ophthalmology Clinic of Ioannina, Greece

Summary: Capsular bag distension syndrome is a very rare complication of cataract surgery. Most cases occur after uneventful phacoemulsification with continuous curvilinear capsulorhexis and implantation of an intraocular lens in the capsular bag. The entity presents with reduction of visual acuity and myopic shift in the early-to-late postoperative period. Characteristic findings include the distension of the capsular bag due to the accumulation of milky material and the forward displacement of the intraocular lens. We present two cases with an unusually delayed presentation of 6 and 8 years respectively following phacoemulsification, and describe their successful management with Nd:YAG laser posterior capsulotomy.

Key words: Capsular block syndrome; YAG laser; Capsulotomy; Capsular bag; Phacoemulsification; Continuous curvilinear capsulorhexis

\section{Introduction}

Capsular bag distension syndrome (CBDS) or capsular block syndrome has been described in patients who have undergone cataract extraction with continuous curvilinear capsulorhexis (CCC) and phacoemulsification with a posterior chamber intraocular lens (IOL) implantation. It is thought to occur due to the occlusion of the CCC opening by the optic of the IOL and the accumulation of whitish opaque fluid in the capsular bag. We report two cases of late onset capsular bag distension syndrome after several years of uneventful phacoemulsification.

\section{Case reports}

\section{Case 1}

A 72-year-old man was referred to our clinic with complains of recently blurred vision and reduction in visual acuity in the right eye (OD). He reported no complains in the left eye (OS). The general medical history was unremarkable and his ophthalmic history was only relevant for uneventful phacoemulsification with posterior chamber IOL implantation eight years ago in OD elsewhere. On examination, best-corrected visual acuity (BCVA) was 5/10 with $-2.00 \mathrm{D}$ in OD and 10/10 without correction in OS. Slit lamp examination revealed deep, quiet anterior chambers without signs of ocular trauma or inflammation. The intraocular pressure was $15 \mathrm{mmHg}$ with Goldmann tonometry in both eyes. Dilated slit lamp examination in OD revealed a distended capsular bag with opaque, whitish fluid between the capsular bag and the posterior surface of the IOL (Figure 1). Additionally, the posterior capsule was opaque. Detailed fundus examination in OD was difficult due to the unclear media but no major pathology could be detected in the posterior pole. The examination of the left eye was unremarkable. $\mathrm{Nd}$ :YAG laser posterior capsulotomy was performed in OD (Figure 2). The fluid flashed to the vitreous cavity through

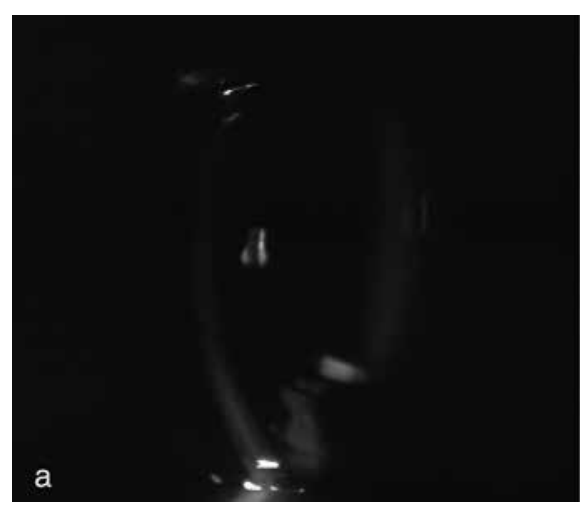

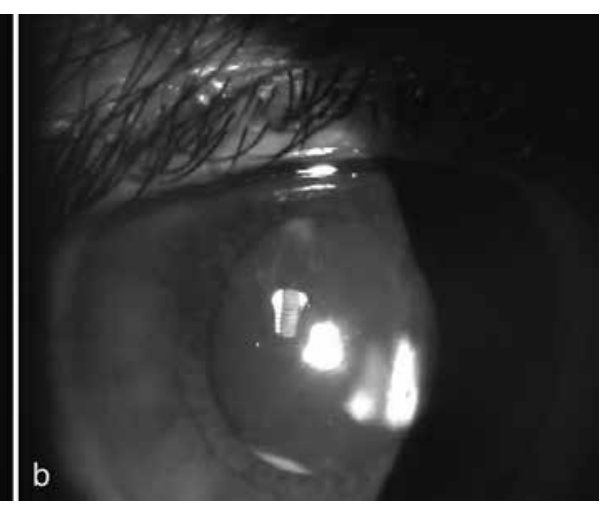

Fig. 1: Slit lamp photographs at presentation. (a) The opaque meniscus of fluid trapped in the space between the intraocular lens and the posterior capsule is evident. (b) View with diffuse slit lamp illumination. 


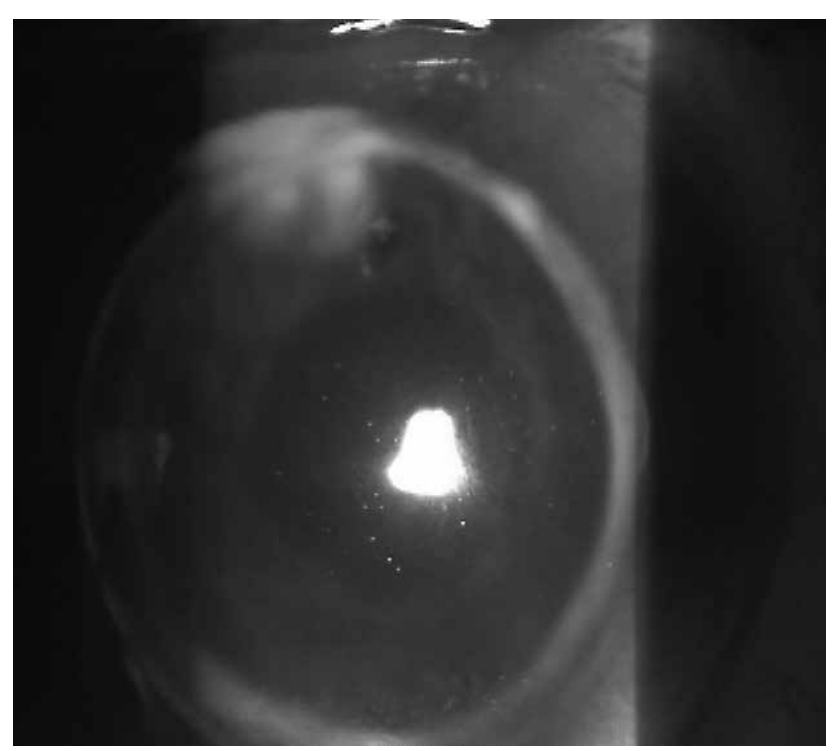

Fig. 2: Slit lamp photograph following Nd:YAG laser capsulotomy.

the capsulotomy opening suggesting a pressure gradient between the IOL/posterior capsule compartment and the vitreous cavity. The patient's visual acuity increased to $6 / 10$ without correction within one hour after Nd:YAG laser capsulotomy and reached 10/10 without correction after a few days. Three months later the vision remained 10/10 without correction.

\section{Case 2}

A 71-years-old Caucasian woman was referred to our clinic with complains of progressively decreased vision in OD. Her general medical history was only significant for arterial hypertension for which she was under medication. Her ophthalmic history was only relevant for uneventful phacoemulsification with posterior chamber IOL implantation in both eyes approximately six years ago elsewhere. She reported no complains in her left eye. On examination, Snellen BCVA was $6 / 10$ with -1.00 D in OD and 10/10 with $-0.50 \mathrm{D}$ in OS. Slit lamp examination showed a normal cornea and an anterior chamber without flare or cells. There were no sings of previous ocular trauma or inflammation. The edge of the anterior CCC was fibrotic and seemed attached to the anterior surface of the IOL optic. The bag was distended and contained a semitransparent fluid with white debris that had precipitated at the lower part. Posterior capsule opacification was also noted. Fundus examination was unremarkable. The intraocular pressure was $12 \mathrm{mmHg}$ in both eyes with Goldmann tonometry. The left eye only had incipient posterior capsule opacification. Nd:YAG laser posterior capsulotomy in OD was performed. Immediately after the perforation of the posterior capsule, the semitransparent fluid was observed to drain fast into the vitreous cavity, indicating the presence of a pressure gradient between the
IOL/posterior capsule compartment and the vitreous cavity. After the drainage of the fluid the capsulotomy was enlarged as clinically indicated. Immediately after the capsulotomy, BCVA increased to $9 / 10$ with $-0.75 \mathrm{D}$. At the one-month post-treatment visit, BCVA was $10 / 10$ without refractive correction and remained so until the last recorded visit six months later.

\section{Discussion}

Capsular bag distension syndrome (CBDS) is a rare complication of phacoemulsification with $\mathrm{CCC}$ but the condition has very rarely been observed after can-opener capsulotomy and extracapsular cataract extraction (1-5). The onset of CBDS varies from a few weeks after cataract surgery to several years. Miyake et all characterised the syndrome according to the time of onset as intraoperative, early postoperative and late postoperative (6). In our cases, the time of CBDS appearance ( 8 and 6 years in the first and second patient respectively) was longer compared to the reported 4-5 years in most reports (3, 6-9). In CBDS, the periphery of the capsulorrhexis is firmly attached to the anterior surface of the IOL so that a closed chamber between the intraocular lens and the posterior lens capsule is created. This chamber is filled with a white and cloudy to milky liquid. Although the precise origin of the liquid remains unknown, it is thought that residual lens epithelial cells undergo metaplastic changes and produce collagen and other extracellular matrix material in the retro-lenticular space. The products of these metaplastic cells create an osmotic gradient that draws aqueous fluid inside the bag, causing its distension (9). Eifrig reported that the aspirated intracapsular fluid from patients with lacteocrumenasia (lacteo: milky, crumen: small bag or coin purse) consisted of alpha-crystalline and albumin. The absence of gamma globulin led the author to the conclusion that the fluid was not the result of an antigen-antibody reaction in the capsular bag (9).

A commonly reported feature of CBDS are changes in refraction. As a result of capsular bag distension, the intraocular lens can move forward causing a myopic shift. This finding was quite characteristic in our cases. Of note, the decompression of the capsular bag lead to an almost immediate reversal of the myopic shift in our patients. Interestingly, a hyperopic shift has also been described and may be explained by the refractive index of the accumulated fluid or the creation of a "minus" lens effect by the accumulated fluid meniscus (10).

A number of treatment choices can be considered in patients with CBDS. Nd:YAG laser capsulotomy of the posterior lens capsule and drainage of the fluid in the vitreous cavity is a frequently suggested option $(10,11)$. Other therapeutic approaches include Nd:YAG laser peripheral iridotomy and anterior capsulotomy (12). With this approach, the turbid intracapsular fluid is drained into the anterior chamber and filtered through the natural outflow system of the eye. An alternative approach is the surgical aspira- 
tion of the fluid through the pars plana $(6,8)$. In our cases, $\mathrm{Nd}$ :YAG laser posterior capsulotomy was very effective in evacuating the opaque, distended capsular bag and allowing the IOL to resume its normal position. It should be noted, however, that the presence of Propionibacterium acnes in the fluid of CBDS cases has been documented (7). To minimize the possibility of Nd:YAG laser-induced Propionibacterium dissemination and endophthalmitis, a pars plana approach with intravitreal injection of antibiotics has been suggested (8). In our cases however, the decision to perform Nd:YAG capsulotomy was based on the total absence of even traces of anterior chamber or vitreous inflammation that could indicate infection by Propionibacterium. Our decision was further supported by the body of existing evidence that this treatment option has so far proved safe, effective and convenient, without the associated risks of a more invasive pars plana approach.

In conclusion, CBDS can occur several years after uneventful phacoemulsification with CCC. Until the role of $\mathrm{Nd}$ :YAG laser capsulotomy and pars plana fluid evacuation is clarified by further research, clinician judgment and careful weighing of potential risks and benefits remain of utmost significance.

\section{References}

1. Davison JA. Capsular bag distension after endophacoemusification and posterior chamber intraocular lens implantation. J Cataract Refract Surg 1990; 16: 99-108.

2. Agrawal S, Agrawal J, Agrawal TP. Complete capsular bag distension syndrome. J Cataract Refract Surg 2000; 26: 1417-1418.

3. Miyake K, Ota I, Miyake S, Horiguchi M. Liquefied aftercataract: a complication of continuous curvilinear capsulorhexis and intraocular lens implantation in the lens capsule. Am J Ophthalmol 1998; 125: 429-35.

4. Starr MB. Liquefied aftercataract: a complication of continuous curvilinear capsulorhexis and intraocular lens implantation in the lens capsule. Am J Ophthalmol 1998; 126: 612-3.

5. Heo JY, Ahn AD, Joo CK. Two cases of late postoperative capsular block syndrome. Korean J Ophthalmol 1999; 13: 105-9.

6. Miyake K, Ota I, Ichihashi S, Miyake S, Tanaka Y, Terasaki H. New classification of capsular block syndrome. J Cataract Refract Surg 1998; 24: 1230-1234.

7. Kollias A, Vogel M, Kaspar H, Lackerbauer C, Grueterich M. Propionibacterium acnes in capsular bag distension syndrome. J Cataract Refract Surg 2010; 36: $167-169$

8. Rana M, Jiang L, Ilango B, Yang YC. Late-onset capsular block syndrome: Unusually delayed presentation. Case Rep Ophthalmol 2013; 4: 299-302.

9. Eifrig DE. Capsulorhexis-related lacteocrumenasia. J Cataract Refract Surg 1997; 23: $450-454$.

10. Wendrix G, Zeyen T. Late-onset capsular bag distention syndrome after cataract surgery. 2 case-reports. Bull Soc Belge Ophthalmol 2006; 32: 351-352.

11. Tan YL, Mohanram LS, Ti SE, Aung T, Perera S. Imaging late capsular bag distension syndrome: an anterior segment optical coherence tomography study. Clin Ophthalmol 2012; 6: 1455-8.

12. Grover DS, Goldberg RA, Ayres B, Fantes F. Treatment of late-onset capsular distension syndrome with a neodymium:YAG laser peripheral iridotomy and anterior capsulotomy. J Cataract Refract Surg 2012; 38: 938-40.

Received: $20 / 09 / 2014$

Accepted in revised form: 25/01/2015

\section{Corresponding author:}

Paraskevas Zafeiropoulos, MD, University Ophthalmology Clinic, Stavros Niarchos Avenue, 45500 Ioannina, Greece; e-mail: pariszafeirop@gmail.com 